\title{
Evidências de Validade de Critério para o Teste de Wartegg
}

\author{
Fernando Pessotto ${ }^{1}$ \\ Centro Universitário Salesiano, Americana-SP, Brasil \\ Ricardo Primi \\ Universidade São Francisco, Campinas-SP, Brasil
}

\section{RESUMO}

O Wartegg é um teste gráfico de autoexpressão, que tem como foco identificar aspectos da personalidade. Com o objetivo de encontrar evidências de validade de critéri, este estudo contou com 40 indivíduos separados igualmente em dois grupos, sendo um composto por esquizofrênicos e o outro, por sujeitos sem histórico de patologia psiquiátrica. Após todos os participantes terem respondido ao Teste de Wartegg, estes foram codificados no sistema proposto por Pessotto (2015). Os resultados indicaram que, das 55 variáveis possíveis analisadas, sete apresentaram diferença significativa entre os grupos. Na regressão logística, foi possível observar o caráter preditivo das variáveis, com destaque para a Má Qualidade Formal (FQ-) e Movimento Humano (M). Para essas duas variáveis, observou-se que seu uso em conjunto é favorável, indicando assim evidências de validade de critério. Ressalta-se que novos estudos são indicados visto que este se trata de um estudo inicial com o sistema.

Palavras-chave: psicometria; avaliação psicológica; técnicas de autoexpressão; esquizofrenia.

\section{ABSTRACT - Evidence of Criterion Validity for the Wartegg Test}

Wartegg is an expressive graphic test focused on identifying aspects of personality. In order to find evidence of criterion validity for the same, this study included 40 individuals, equally divided into two groups, one consisting of schizophrenics and the other of subjects with no history of psychiatric pathology. After all participants had responded to the Wartegg Test, they were coded in the system proposed by Pessotto (2015). Results indicated that, of the 55 possible variables analyzed, 7 presented a significant difference between the groups. In the logistic regression it was possible to observe the predictive character of the variables with emphasis on poor Formal Quality (FQ) and Human Movement (M). For these two variables, combined use was observed as favorable, indicating evidence of criterion validity. Emphasis is given to the need for further research as this is an initial study with the system. Keywords: psychometrics; psychological evaluation; self-expression techniques; schizophrenia.

\section{RESUMEN - Evidencias de Validez de Criterio para el Test de Warteeg}

El test de Wartegg es un test gráfico expresivo, que tiene como objetivo primordial identificar aspectos de la personalidad. Con motivo de encontrar evidencia de validez de criterio para el mismo, el estudio se realizó a 40 personas. Estas fueron separadas en dos grupos. El primer grupo, fue formado por pacientes diagnosticados con esquizofrenia, mientras que el segundo, lo conformaron personas que no poseían ningún histórico de patologías psiquiátricas. Tras finalizar todos los participantes el test de Wartegg, los resultados fueron codificados de acuerdo al sistema propuesto por Pessotto (2015). Estos resultados indicaron que, de las 55 variables posibles analizadas, 7 presentaron diferencia significativa entre los grupos. En la regresión logística fue posible observar el carácter predictivo de las variables con destaque para la Mala Calidad Formal (FQ-) y Movimiento Humano (M). Para estas dos variables se observó que su uso de forma conjunta es favorable, indicando, así, evidencias de validez de criterio. Se puede resaltar que nuevas investigaciones pueden ser recomendadas, puesto que se trata de un estudio inicial con el sistema.

Palabras clave: psicometría; evaluación psicológica; técnicas de autoexpresión; esquizofrenia.

As técnicas de autoexpressão fazem parte de um grupo de instrumentos psicológicos caracterizados por utilizarem estímulos pouco estruturados com o intuito de favorecer a livre expressão do indivíduo durante o processo de avaliação (Fensterseifer \& Werlang, 2008; Meyer \& Kurtz, 2006). Por meio dessas técnicas, busca-se interpretações que possam ser indicadores do tipo de funcionamento característico do indivíduo, portanto, de acordo com o comportamento e desempenho do sujeito durante a prova infere-se um modo interno de funcionamento podendo este ser classificado em diferentes tipos, como extrovertido, sociável, estratensivo, entre 
outros (Fensterseifer \& Werlang, 2008; Villemor-Amaral \& Pasqualini-Casado, 2006).

Nessas técnicas, essas classificações são organizadas de acordo com indicadores presentes nos sistemas de correção, como tamanho de imagem, posição, cores, entre outros, a partir da interação do sujeito com o estímulo, podendo ser manchas de tinta, histórias, desenhos e, devido o fato de serem pouco estruturadas, apresentam menor possibilidade de manipulação consciente sobre as respostas (Werlang \& Cunha, 1993). Nesse sentido, a organização do campo visual, envolvendo desde mecanismos básicos de percepção até instâncias mais complexas como focos de interesse e atribuição de significados, são indicadores do tipo de funcionamento que o indivíduo apresenta (Koffka, 1975).

Nessa perspectiva, o indivíduo é concebido como uma totalidade, não representando apenas um somatório de variáveis ou traços latentes que o compõem e confere um sentido particular sobre o estímulo sem significado. Sendo assim, seja nas histórias, desenhos, estrutura das frases expressas, ou ainda na associação de manchas, a qualidade estrutural do que está sendo projetado demonstra o nível de organização dos processos mentais, indicando instabilidades e estabilidade, rigidez e flexibilidade, apresentando assim o grau de patologia ou saúde de seu mundo interior, baseado em sua percepção (Kolck, 1974; Montagna, 1989; Villemor-Amaral, 2008).

O processo de percepção é a maneira que a experiência é percebida, assimilada e interpretada subjetivamente pelo sujeito (Werlang, 2002), sendo concebida num continuum indo da percepção objetiva até a apercepção, ou seja, a distorção perceptiva da realidade. Esse processo se dá de acordo com um princípio gestáltico em que o todo é mais que a soma das partes, nesse caso, indicando que não é possível classificar o grau de percepção geral do sujeito, mas de que forma as diferentes nuances perceptivas interagem caracterizando assim um sistema de relações perceptivas (Koffka, 1975; Silva, 1989). Montagna (1989) salienta que não se deve interpretar a expressão observada como o comportamento real do sujeito, mas como necessidades ou fantasias.

É possível verificar a complexidade desse tipo de avaliação muitas vezes tida como subjetiva. Devido essas características, as técnicas de autoexpressão sofrem frequentes críticas no que se refere aos parâmetros psicométricos, como normas, evidências de validade e fidedignidade (Garb, Wood, Lilienfeld, \& Nezworski, 2002; Lilienfeld, Wood, \& Garb, 2000; Villemor-Amaral \& Werlang, 2008). Contudo, Lililenfeld et al. (2000) apontam que essa é uma concepção falha e que são necessários outros métodos de estudo devido às diferenças substanciais na operacionalização das tarefas e construção de escalas de autorrelato e de autoexpressão.

Villemor-Amaral e Pasqualini-Casado (2006) completam dizendo que os parâmetros empregados na análise de técnicas de autoexpressão devem considerar o raciocínio clínico com foco idiográfico que enfatiza o indivíduo, diferentemente do foco nomotético, que tem como foco regras gerais comuns em determinados grupos. Portanto, conforme indica Campos (2013), a rede nomotética busca verificar semelhanças entre o sujeito em comparação com a população, enquanto a idiográfica, a forma que o sujeito se difere da maioria das pessoas, indicando características particulares de seu funcionamento.

Sobre essa diferença, alguns autores (Bornstein, 2001, 2002; Meyer \& Handler, 1997; Villemor-Amaral \& Pasqualini-Casado, 2006; Wilson, Lindsey, \& Schooler, 2000) relatam que medidas de autorrelato e de autoexpressão acessam informações acerca de variáveis psicológicas de forma diferenciada, mesmo quando são concebidos a medir construtos semelhantes. Isso pode implicar em baixas correlações entre as medidas, podendo, portanto, não indicar um problema de evidências de validade convergente, mas sustentar, justamente, que o acesso ao construto foi feito de modo diferenciado, resultando numa medida variada. Outros autores (Baumeister \& Tice, 1988; Chaplin, 1991; Swann \& Seyle, 2005) afirmam ainda que essas correlações não são constantes, mas variáveis de acordo com fatores, como frequência e intensidade dos traços, ou ainda o contexto de mensuração.

No entanto, algumas técnicas de autoexpressão ainda carecem de bons sistemas de correção e interpretação. Essa situação, além de desfavorecer o objetivo de um processo de avaliação psicológica, pode inviabilizar definitivamente o uso de algumas técnicas. Especificamente no Brasil, o Conselho Federal de Psicologia (CFP, 2003) estabeleceu critérios mínimos referentes a evidências de validade e precisão, para indicar parecer de uso favorável ou não de testes psicológicos a fim de garantir fundamentação científica suficiente para embasar as interpretações feitas a partir dos resultados coletados. Com isso, algumas técnicas de autoexpressão, como o Teste das Fábulas (Cunha \& Nunes, 1993) e o Wartegg (Freitas, 1993), por exemplo, tiveram, no Brasil, parecer desfavorável ao uso pelo CFP, devido não terem apresentado os critérios mínimos para utilização.

No que se refere ao contexto desfavorável acerca das evidências de validade do Teste Wartegg, Roivainen (2009) salienta que a criação de critérios mais claros e objetivos para a atribuição dos códigos poderia favorecer melhores índices de evidências de validade, não limitando à necessidade de interpretações baseadas apenas em características idiográficas, muitas vezes, de caráter subjetivo. Essa consideração é complementada por Gronnerod e Gronnerod (2012), salientando a importância da ampliação dos estudos com o objetivo de desenvolver novos parâmetros para a técnica.

Alves (2008) realizou uma revisão sistemática sobre pesquisas realizadas com o instrumento no Brasil, compreendendo em quatro grupos, sendo eles, temas diversos, estudos normativos, de precisão e de evidências de 
validade. Para temas diversos, foram consideradas pesquisas que utilizaram o Teste de Wartegg em diferentes contextos, sendo encontrados 21 publicados no período de 1994 a 2003. Destes, um refere-se a um relato de experiência, dois são dissertações, um capítulo de livro e dezessete são trabalhos apresentados em congressos ou encontros científicos, carecendo de informações aprofundadas sobre métodos e até mesmo resultados. Alguns destes apresentavam conclusões sobre aspectos psicológicos avaliados pelo Wartegg, mas pelo fato de, em sua maioria, terem sido encontrados apenas os resumos, estes não indicavam a partir de que dados ou variáveis foram evidenciados, o que torna difícil a compreensão dos resultados obtidos.

No que se refere aos estudos normativos, foram encontrados dois, sendo uma dissertação de mestrado e uma tese de doutorado. Sobre a precisão foram encontrados dois, sendo um referente a um manuscrito não publicado e uma dissertação de mestrado. Finalmente, sobre os estudos de evidências de validade foram apresentados quatro, sendo dois apenas resumos apresentados em congressos e duas dissertações de mestrado. A autora conclui que, embora a literatura não apresente grande quantidade de trabalhos, alguns dados permitem compreender as qualidades psicométricas do Teste de Wartegg e, sobretudo, orientações para futuros estudos (Silva, 2008).

Outro levantamento bibliográfico com resultados semelhantes foi realizado por Roivainen (2009), que verificou, em uma pesquisa realizada à base de dados PsycInfo, 88 estudos utilizando o Teste de Wartegg, no período de 1930 a 2006. Essa quantidade de trabalhos é pequena, considerando sua data de criação e também em comparação com outros testes de autoexpressão, como o Rorschach no Sistema Compreensivo, por exemplo, que, no período de 1940 a 2006, foram publicados mais de 9000 trabalhos. Roivainen (2009) ainda complementa que a maior parte dos artigos é de base teórica e, nos poucos estudos empíricos, os índices de evidências de validade e precisão são baixos ou não satisfatórios.

Esses achados são corroborados por Gronnerod e Gronnerod (2012) que, ao realizarem uma revisão sobre o uso do Teste de Wartegg, verificaram primeiramente a baixa quantidade de estudos, sendo a maior parte deles teóricos, e, da mesma forma que os autores anteriores, apresentam índices não satisfatórios de evidências de validade e precisão. Outra constatação feita foi a inexistência de um sistema internacional de correção e interpretação, dificultando estudos transculturais, o que poderia auxiliar com mais fontes de evidências de validade.

Ramon (2006) realizou um estudo de evidências de validade concorrente do Teste de Wartegg com o Rorschach-SC contando com 40 sujeitos com idades variando entre 18 e 55 anos. A partir da codificação do Teste de Wartegg foram estabelecidas quatro variáveis de acordo com os critérios diagnósticos do teste, sendo elas, relacionamento interpessoal, afetividade e controle emocional, ambição e adaptação a normas e regras, sendo utilizados os mesmos indicadores para o Rorschach-SC. Os resultados indicaram correlação de alta magnitude para afetividade e controle emocional $(0,84)$, mediana para relacionamento pessoal $(0,59)$, baixa para adaptação a normas $(0,33)$ e correlação não significativa para ambição indicando um resultado insatisfatório e a falta de evidências de validade convergente com os indicadores do Rorschach-SC. O autor relata a necessidade de novos estudos que busquem definir parâmetros mais precisos em relação à classificação e interpretação e finaliza indicando a necessidade de definição das características que o teste se propõe a avaliar, pois foram encontradas divergências na conceituação.

Souza, Primi e Miguel (2007) também verificaram algumas inconsistências no Teste de Wartegg referente a índices de evidências de validade, mesmo tendo sido um instrumento amplamente utilizado por psicólogos no Brasil. Contando com uma amostra de 121 sujeitos com idade entre 16 e 65 anos, os autores buscaram examinar as evidências de validade convergente, comparando-o aos instrumentos 16PF, BPR-5 e o Questionário de Avaliação de Desempenho. Para essa análise foram criadas 141 variáveis para o Teste de Wartegg segundo as hipóteses interpretativas sugeridas por Freitas (1993).

Em relação ao $16 \mathrm{PF}$, foram encontradas algumas correlações positivas e outras negativas, porém nem todas apresentam coerência com a hipótese interpretativa do Teste de Wartegg. No Fator F (sobriedade e despreocupação), por exemplo, foi verificada uma correlação positiva significativa com a presença de esquematismo do Wartegg $(r=0,27 ; p<0,003)$, contrariando a hipótese de que esse indicador mostraria dificuldades em relacionamentos interpessoais, já que, quanto maior a intensidade dos conteúdos esquemáticos, o sujeito apresentaria também maior alegria e despreocupação. Ainda referente ao Fator F, eram esperadas correlações com as variáveis do Teste de Wartegg que indicam aspectos de comunicação, espontaneidade e relacionamento com outras pessoas, o que não foi indicado pelas análises (Souza, Primi, \& Miguel, 2007).

Para a BPR-5, Souza et al. (2007) verificaram correlações positivas significativas para a variável expansão interrompida para os Quadros 4, 5, 7 e $8(r=0,23$ a $r=0,31)$. As análises com as provas separadas indicaram correlação positiva $(r=0,21 ; p<0,016)$ da prova de raciocínio verbal com a escolha do Quadro 6 como preferido. A escolha desse Quadro como o preferido pode indicar invasão de fantasia no pensamento lógico, ligado a um funcionamento mais subjetivo. Enfim, para o Questionário de Avaliação de Desempenho, foram verificadas correlações de magnitude baixa, tanto positivas quando negativas, ressaltando a correlação positiva entre o desempenho e a variável de excesso de linhas curvas $(r=0,24 ; p<0,011)$, indicando emotividade, flexibilidade e capacidade de ajustamento. 
Em síntese, os autores apontam que, das 141 variáveis analisadas, em relação aos fatores do $16 \mathrm{PF}$ e da BPR5 , foram verificadas 13 correlações coerentes de acordo com o esperado, seis incoerentes e três neutras. Em relação ao desempenho, apenas seis apresentaram correlação significativa, sendo três delas de baixa magnitude. Os autores concluem que muitas das interpretações teóricas realizadas no teste não apresentam correlação com os resultados dos outros testes que avaliam construtos semelhantes, indicando inconsistência nas inferências realizadas a partir delas (Souza et al., 2007).

Nesse sentido, considerando que instrumentos indicados para a avaliação da personalidade são de grande importância no acompanhamento e compreensão de patologias, o presente trabalho teve como objetivo a verificação de evidências de validade de critério para Teste de Wartegg segundo o sistema proposto por Pessotto (2015). Considerando as proposições feitas acerca de percepção e a relação destas com as técnicas de autoexpressão, tem-se por hipótese que os indicadores que apresentarem maior poder diagnóstico diferenciarão sujeitos diagnosticados com esquizofrenia visto que essa patologia tem impacto direto na percepção do indivíduo. Conforme apontam Gronnerod e Gronnerod (2012), é necessária a construção de sistemas sólidos, pautados em grande quantidade de estudos, gerando conhecimento acumulado para utilização de técnicas de avaliação psicológica.

\section{Método}

\section{Participantes}

Participaram deste estudo 40 sujeitos, divididos em dois grupos. O grupo 1 foi composto por 20 pacientes com diagnóstico de esquizofrenia provenientes de duas instituições, à saber, um hospital público psiquiátrico localizado no interior de São Paulo e um centro de saúde público com atendimentos voltados a essa população. Para o grupo 2, procedeu-se o pareamento dos sujeitos, considerando a faixa etária aceitando um desvio padrão de cinco anos, etnia, estado civil, sexo e escolaridade, selecionados via mídias sociais, instituições de ensino e organizações.

Sobre a totalidade dos participantes, a idade variou de 21 a 70 anos $(M=40 ; D P=12,8)$, sendo 36 do sexo masculino. Acerca da etnia, 28 eram brancos, seis negros e seis declararam-se de outras etnias. No que diz respeito à escolaridade, ela variou de fundamental incompleto à pós-graduação conforme apresentado na Tabela 1.

Tabela 1

Distribuição das Escolaridades dos Participantes

\begin{tabular}{lcc}
\hline & Frequência & Porcentagem \\
\hline Fundamental incompleto & 14 & $35 \%$ \\
Fundamental completo & 10 & $25 \%$ \\
Ensino médio incompleto & 8 & $20 \%$ \\
Ensino médio completo & 2 & $5 \%$ \\
Superior incompleto & 2 & $5 \%$ \\
Superior completo & 2 & $5 \%$ \\
Pós-graduação & 2 & $5 \%$ \\
Total & 40 & $100 \%$ \\
\hline
\end{tabular}

\section{Instrumento}

Teste de Wartegg (Pessotto, 2015). O Teste de Wartegg é um teste gráfico de autoexpressão, semiestruturado, que tem como objetivo identificar aspectos da personalidade. O método utiliza uma folha de estímulos composta por oito quadrados de $4 \mathrm{~cm} \mathrm{x} 4 \mathrm{~cm}$, divididos por uma moldura preta de $6 \mathrm{~mm}$. Cada um contém um estímulo impresso, sendo solicitado ao sujeito continuar o desenho, formando uma única figura para cada quadrado, a partir desses estímulos impressos. Em seguida, é realizado um inquérito sobre as figuras contendo questões, como o desenho que mais agradou e que menos agradou, ou ainda, os estímulos que agradaram mais ou menos. A aplicação dura cerca de 15 minutos.

No sistema proposto por Pessotto (2015), são utilizadas as variáveis conforme indicado na Tabela 2. Utilizando esse sistema de codificação. Pessotto (2015) verificou boa concordância entre juízes com índices de correlação, variando de 0,53 a 0,94 entre os protocolos corrigidos por quatro juízes participantes do estudo. Contudo o coeficiente kappa para as variáveis anteriormente apresentadas do Wartegg não demonstraram-se satisfatórios, variando entre 0,05 e 0,69 , indicando necessidade de outros estudos para ajuste do sistema de codificação. 
Tabela 2

Variáveis do Wartegg Utilizadas no Sistema Proposto por Pessotto (2005)

\begin{tabular}{|c|c|c|}
\hline $\begin{array}{c}\text { Variável } \\
\text { Orientação }\end{array}$ & \multicolumn{2}{|l|}{ Subdivisão } \\
\hline \multirow{3}{*}{ Localização } & Vertical & $\begin{array}{l}\text { superior } \\
\text { inferior }\end{array}$ \\
\hline & horizontal & $\begin{array}{l}\text { esquerda } \\
\text { direita }\end{array}$ \\
\hline & $\begin{array}{l}\text { central } \\
\text { Total }\end{array}$ & \\
\hline Tamanho & $\begin{array}{l}\text { pequeno } \\
\text { médio } \\
\text { grande }\end{array}$ & \\
\hline Conteúdos & $\begin{array}{l}\text { figura humana inteira } \\
\text { figura para-humana inteira } \\
\text { parte de figura humana } \\
\text { parte de figura pára-humana } \\
\text { animal inteiro } \\
\text { parte de animal } \\
\text { parte de pára-animal } \\
\text { anatomia } \\
\text { arte } \\
\text { antropologia } \\
\text { sangue } \\
\text { vestuário } \\
\text { explosão } \\
\text { fogo } \\
\text { sexo } \\
\text { conteúdo que não se enquadra em nenhum dos anteriores }\end{array}$ & \\
\hline Qualidade dos Objetos & $\begin{array}{l}\text { síntese } \\
\text { vago }\end{array}$ & \\
\hline Qualidade Formal & $\begin{array}{l}\text { ordinário (boa qualidade formal, e frequente) } \\
\text { incomum (boa qualidade formal, mas infrequente) } \\
\text { distorcido (má qualidade formal) }\end{array}$ & \\
\hline Popular & & \\
\hline & movimento humano & \\
\hline & movimento animal & $\begin{array}{l}\text { ativo } \\
\text { passivo }\end{array}$ \\
\hline Caracterıstıcas Partıculares & $\begin{array}{l}\text { profundidade (sombreado) } \\
\text { textura (sombreado) } \\
\text { profundidade }\end{array}$ & \\
\hline Códigos Especiais & $\begin{array}{l}\text { combinação fabulada } \\
\text { contaminação } \\
\text { representação abstrata } \\
\text { movimento cooperativo } \\
\text { mórbido } \\
\text { mutualidade de autonomia (saudável) } \\
\text { mutualidade de autonomia (patológico) } \\
\text { boa representação humana } \\
\text { má representação humana } \\
\text { movimento agressivo } \\
\text { conteúdo agressivo } \\
\text { linguagem dependente }\end{array}$ & \\
\hline
\end{tabular}


Tabela 2 (continuação)

Variáveis do Wartegg Utilizadas no Sistema Proposto por Pessotto (2005)

\begin{tabular}{|c|c|c|}
\hline Variável & \multirow{2}{*}{\multicolumn{2}{|c|}{ Subdivisão }} \\
\hline Orientação & & \\
\hline \multirow{7}{*}{ Tipo de Traço } & & fraco \\
\hline & pressão & moderado \\
\hline & & forte \\
\hline & & contínua \\
\hline & & descontínua \\
\hline & linha & trêmula \\
\hline & & reforçada \\
\hline \multirow{2}{*}{ Repetição } & duplicação & \\
\hline & fechamento & \\
\hline \multirow{6}{*}{ Análise de Sequência } & rígida & \\
\hline & ordenada & \\
\hline & invertida rígida & \\
\hline & invertida indireta & \\
\hline & rigida atípica & \\
\hline & relaxada & \\
\hline Título & & \\
\hline
\end{tabular}

\section{Procedimentos}

Primeiramente o projeto foi aprovado pelo Comitê de Ética em Pesquisa da Universidade São Francisco. Depois dessa aprovação, os sujeitos foram convidados a participar do estudo, assinando o Termo de Consentimento Livre e Esclarecido (TCLE). Conforme a resolução 196/96 do Conselho Nacional de Saúde, o próprio participante pode assinar o TCLE, salvo nas situações em que ele apresentar capacidade de autodeterminação reduzida. Nesses casos, foi solicitado a um responsável legal a assinatura do TCLE.

As aplicações foram realizadas de forma individual em locais disponibilizados pelos campos de coleta (hospital psiquiátrico, centro de saúde, serviço escola, empresas e clínica particular). Primeiramente, a coleta foi realizada com os pacientes com esquizofrenia e, posteriormente, tendo por base o perfil socioeconômico deles, com o grupo normativo sem intervalo de tempo entre as aplicações. Todos os sujeitos responderam ao Teste de Wartegg com duração média de 15 minutos.

\section{Análise de Dados}

Inicialmente empregou-se o teste $t$ de Student com a finalidade de identificar as diferenças entre as variáveis para os dois grupos, seguido do $d$ de Cohen, com o objetivo de verificar diferenças significativas para cada um deles. Espera-se, sobretudo, encontrar tais diferenças para as variáveis referentes à qualidade formal $(\mathrm{FQu}$ e FQ-). Esses códigos são atribuídos de forma similar ao Rorschach-SC em que FQu indicaria boa adequação do desenho confeccionado ao estímulo inicial e FQ-, o contrário. Em resumo, a boa qualidade formal indica que o estímulo inicial presente no quadro foi utilizado de forma harmoniosa ao desenho executado. Feito isso, empregou-se a regressão logística para as variáveis com diferenças significativas entre os grupos, agrupando-as de acordo com os critérios de classificação do sistema de Pessotto (2015). Para essas variáveis, foi utilizado o procedimento Curva ROC para verificar a capacidade de acertos diagnósticos no uso do instrumento, bem como a sensibilidade e especificidade com o objetivo de verificar a acurácia diagnóstica do sistema proposto para o Teste de Wartegg.

\section{Resultados e Discussão}

Com o objetivo de verificar evidências de validade de critério para o conjunto de variáveis proposto por Pessotto (2015) para o Teste de Wartegg, empregou-se o teste $t$ de Student bem como o $d$ de Cohen para verificar a diferença entre o grupo de esquizofrênicos e normativos. As comparações entre os grupos foram executadas para cada uma das variáveis. Esses índices podem ser observados na Tabela 3 .

É possível observar que, das 55 variáveis analisadas, sete apresentaram diferença significativa entre os grupos, a saber, boa qualidade formal (FQu), má qualidade formal (FQ-), movimento humano (M), movimento animal (FM), movimento passivo (p), tipo de traço - pressão fraca (Pres_fra), sequência ordinária (Seq_ord). Conforme hipótese citada anteriormente, verificou-se diferença significativas para os códigos de qualidade formal, além delas, e três códigos de movimento, um de pressão do traço e sequência. Sendo assim, a hipótese de que os indicadores com maior poder diagnóstico para a esquizofrenia pudessem diferenciar os grupos foi confirmada. 
Tabela 3

Estatísticas Descritivas, Teste t de Student e d de Cohen para os Grupo de Esquizofrênicos (EQ) e Normativo (N)

\begin{tabular}{|c|c|c|c|c|c|c|c|}
\hline Var. & Grupo & $M$ & $\mathrm{DP}$ & $\mathrm{N}$ & $t$ & $p$ & $d$ \\
\hline \multirow{2}{*}{ Loc_sup } & EQ & 2,7 & 2,56 & 20 & \multirow{2}{*}{1,249} & \multirow{2}{*}{0,219} & \multirow{2}{*}{$-0,39$} \\
\hline & $\mathrm{N}$ & 1,9 & 1,29 & 20 & & & \\
\hline \multirow{2}{*}{ Loc_inf } & EQ & 3,65 & 2,58 & 20 & \multirow{2}{*}{1,249} & \multirow{2}{*}{0,463} & \multirow{2}{*}{$-0,23$} \\
\hline & $\mathrm{N}$ & 3,15 & 1,57 & 20 & & & \\
\hline \multirow{2}{*}{ Loc_esq } & EQ & 3,35 & 2,46 & 20 & \multirow{2}{*}{0,848} & \multirow{2}{*}{0,402} & \multirow{2}{*}{$-0,27$} \\
\hline & $\mathrm{N}$ & 2,8 & 1,54 & 20 & & & \\
\hline \multirow{2}{*}{ Loc_dir } & EQ & 3,5 & 2,19 & 20 & \multirow{2}{*}{0,682} & \multirow{2}{*}{0,500} & \multirow{2}{*}{$-0,22$} \\
\hline & $\mathrm{N}$ & 3,1 & 1,45 & 20 & & & \\
\hline \multirow{2}{*}{ Loc_cen } & EQ & 6,35 & 2,35 & 20 & \multirow{2}{*}{0,291} & \multirow{2}{*}{0,772} & $-0,09$ \\
\hline & $\mathrm{N}$ & 6,15 & 1,98 & 20 & & & $-0,0 Y$ \\
\hline & EQ & 1,8 & 2,29 & 20 & & & \\
\hline LOC_tot & $\mathrm{N}$ & 1,6 & 2,09 & 20 & 0,289 & $0, / / 4$ & $-0,09$ \\
\hline ТиmРn & EQ & 2,65 & 2,68 & 20 & 0194 & 0847 & (חم \\
\hline Iantreq & $\mathrm{N}$ & 2,5 & 2,19 & 20 & 0,194 & $0,84 /$ & $-0,06$ \\
\hline TamMed & EQ & 3,2 & 2,19 & 20 & -0.956 & 0.345 & 0.30 \\
\hline I aninved & $\mathrm{N}$ & 3,85 & 2,11 & 20 & & & \\
\hline Tam Gra & EQ & 1,9 & 2,53 & 20 & 0421 & 0676 & 12 \\
\hline lamsra & $\mathrm{N}$ & 1,6 & 1,93 & 20 & $0,4 \angle 1$ & $0,6 / 6$ & $-0,13$ \\
\hline ContH & EQ & 0,05 & 0,22 & 20 & -0831 & 0411 & 026 \\
\hline Lonth & $\mathrm{N}$ & 0,15 & 0,49 & 20 & $-0,831$ & 0,411 & 0,26 \\
\hline Cont(H) & EQ & 0,45 & 1,57 & 20 & 1127 & 0267 & - 36 \\
\hline Conl1(1) & $\mathrm{N}$ & 0,05 & 0,22 & 20 & 1,121 & $0,20 /$ & $-0,36$ \\
\hline ContHd & EQ & 0,45 & 0,76 & 20 & -0873 & 0388 & 028 \\
\hline contha & $\mathrm{N}$ & 0,7 & 1,03 & 20 & $-0,813$ & 0,380 & 0,28 \\
\hline Cont(Hd) & EQ & 0,1 & 0,45 & 20 & -1515 & 0138 & 0.48 \\
\hline & $\mathrm{N}$ & 0,35 & 0,59 & 20 & & & \\
\hline Conta & EQ & 0,2 & 0,52 & 20 & -0326 & 0746 & 010 \\
\hline contA & $\mathrm{N}$ & 0,25 & 0,44 & 20 & $-0,326$ & 0,146 & 0,10 \\
\hline ContAd & EQ & 0,15 & 0,37 & 20 & 1831 & 0075 & 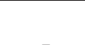 \\
\hline contAa & $\mathrm{N}$ & 0 & 0,00 & 20 & 1,831 & $0,0 / 3$ & - \\
\hline Cont(Ad) & EQ & 0,05 & 0,22 & 20 & 1 & 0.324 & $-0,32$ \\
\hline cont(Au) & $\mathrm{N}$ & 0 & 0,00 & 20 & 1 & 0,324 & $-0,32$ \\
\hline ContAn & EQ & 0,05 & 0,22 & 20 & 1 & 0324 & -032 \\
\hline contAn & $\mathrm{N}$ & 0 & 0,00 & 20 & 1 & $0,3<4$ & $-0,32$ \\
\hline ContArt & EQ & 0,05 & 0,22 & 20 & 1 & 0324 & -032 \\
\hline & $\mathrm{N}$ & 0 & 0,00 & 20 & & & \\
\hline ContAy & EQ & 0 & 0,00 & 20 & $18<-18$ & ( & 050 \\
\hline contAy & $\mathrm{N}$ & 0,15 & 0,37 & 20 & -1,831 & $0,0 / 5$ & 0,58 \\
\hline ContCg & EQ & 0,05 & 0,22 & 20 & 0 & 1000 & 0.00 \\
\hline contcy & $\mathrm{N}$ & 0,05 & 0,22 & 20 & 0 & 1,000 & 0,00 \\
\hline ContFi & EQ & 0,05 & 0,22 & 20 & 0 & 1.000 & 0.00 \\
\hline & $\mathrm{N}$ & 0,05 & 0,22 & 20 & & & \\
\hline ContNC & EQ & 6,10 & 2,00 & 20 & -0673 & 0505 & 021 \\
\hline ContIV & $\mathrm{N}$ & 6,45 & 1,19 & 20 & $-0,6 / 3$ & 0,5us & 0,21 \\
\hline OO Sy & EQ & 0,10 & 0,31 & 20 & -1778 & 0083 & 056 \\
\hline vu_sy & $\mathrm{N}$ & 0,65 & 1,35 & 20 & $-1,1 / 8$ & 0,083 & 0,50 \\
\hline $\mathrm{OO} \mathrm{Vg}$ & EQ & 0,90 & 2,02 & 20 & 1778 & ר & 055 \\
\hline ve_vg & $\mathrm{N}$ & 0,10 & 0,31 & 20 & $1, / / 8$ & 0,089 & $-0,55$ \\
\hline FOu & EQ & 4,15 & 3,53 & 20 & -3794 & 0001 & 120 \\
\hline & $\mathrm{N}$ & 7,45 & 1,64 & 20 & $-3,194$ & 0,001 & 1,20 \\
\hline FQ- & EQ & 3,65 & 3,63 & 20 & 3,56 & 0,001 & $-1,13$ \\
\hline & $\mathrm{N}$ & 0,50 & 1,57 & 20 & & & \\
\hline
\end{tabular}


Tabela 3 (continuação)

Estatísticas Descritivas, Teste t de Student e d de Cohen para os Grupo de Esquizofrênicos (EQ) e Normativo (N)

\begin{tabular}{|c|c|c|c|c|c|c|c|}
\hline Var. & Grupo & $M$ & $D P$ & $\mathrm{~N}$ & $t$ & $p$ & $d$ \\
\hline \multirow{2}{*}{ CP_M } & EQ & 0,10 & 0,45 & 20 & \multirow{2}{*}{$-3,126$} & \multirow{2}{*}{0,003} & \multirow{2}{*}{0,99} \\
\hline & $\mathrm{N}$ & 0,70 & 0,73 & 20 & & & \\
\hline \multirow{2}{*}{ CP_FM } & EQ & 0,00 & 0,00 & 20 & \multirow{2}{*}{$-2,179$} & \multirow{2}{*}{0,036} & \multirow{2}{*}{0,69} \\
\hline & $\mathrm{N}$ & 0,20 & 0,41 & 20 & & & \\
\hline \multirow{2}{*}{ CP_m } & EQ & 0,35 & 0,67 & 20 & \multirow{2}{*}{0,515} & \multirow{2}{*}{0,609} & \multirow{2}{*}{$-0,16$} \\
\hline & $\mathrm{N}$ & 0,25 & 0,55 & 20 & & & \\
\hline \multirow{2}{*}{ CP_a } & EQ & 0,45 & 0,89 & 20 & \multirow{2}{*}{$-0,15$} & \multirow{2}{*}{0,881} & \multirow{2}{*}{0,05} \\
\hline & $\mathrm{N}$ & 0,50 & 1,19 & 20 & & & \\
\hline \multirow{2}{*}{ CP_p } & EQ & 0,00 & 0,00 & 20 & \multirow{2}{*}{$-4,333$} & \multirow{2}{*}{0,000} & 137 \\
\hline & $\mathrm{N}$ & 0,65 & 0,67 & 20 & & & 1,ב \\
\hline$C \mathrm{P} Y$ & EQ & 0,15 & 0,49 & 20 & -4332 & 0168 & 0.44 \\
\hline$C P_{-} r$ & $\mathrm{~N}$ & 0,50 & 1,00 & 20 & $-4,333$ & 0,168 & 0,44 \\
\hline$C \mathrm{P} F \mathrm{~F}$ & EQ & 0,45 & 0,89 & 20 & 1206 & 0235 & 38 \\
\hline 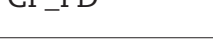 & $\mathrm{N}$ & 0,15 & 0,67 & 20 & 1,८U6 & 0,235 & $-0,38$ \\
\hline$C F$ FAR & EQ & 0,05 & 0,22 & 20 & 1 & 0324 & -032 \\
\hline CE_FAB & $\mathrm{N}$ & 0,00 & 0,00 & 20 & 1 & $0,3<4$ & $-0,32$ \\
\hline$C F \mathrm{CON}$ & EQ & 0,05 & 0,22 & 20 & 1 & 0324 & -032 \\
\hline CE_CUN & $\mathrm{N}$ & 0,00 & 0,00 & 20 & 1 & $0,3<4$ & $-0,32$ \\
\hline$C E A B S$ & EQ & 0,70 & 1,90 & 20 & 0.097 & 0.923 & -0.03 \\
\hline & $\mathrm{N}$ & 0,65 & 1,31 & 20 & & & \\
\hline$C F$ PFR & EQ & 0,10 & 0,31 & 20 & -0794 & 0432 & 025 \\
\hline CE_PER & $\mathrm{N}$ & 0,25 & 0,79 & 20 & $-0, / 94$ & 0,432 & 0,25 \\
\hline$C F M O R$ & EQ & 0,00 & 0,00 & 20 & 1821 & ( & 058 \\
\hline 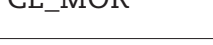 & $\mathrm{N}$ & 0,15 & 0,37 & 20 & $-1,831$ & $0,0 / 3$ & 0,58 \\
\hline CE GHR & EQ & 0,40 & 1,57 & 20 & 0.136 & 0.893 & -0.04 \\
\hline & $\mathrm{N}$ & 0,35 & 0,49 & 20 & & & \\
\hline$C F$ AGM & EQ & 0,05 & 0,22 & 20 & 0 & 1000 & (חم \\
\hline CE_AGIVI & $\mathrm{N}$ & 0,05 & 0,22 & 20 & 0 & 1,000 & 0,00 \\
\hline CF ODI & EQ & 0,15 & 0,49 & 20 & 1371 & 0178 & -043 \\
\hline & $\mathrm{N}$ & 0,00 & 0,00 & 20 & $1,3 / 1$ & & $-0,43$ \\
\hline Pres fra & EQ & 0,10 & 0,31 & 20 & -2444 & 0019 & ( 77 \\
\hline Pres_Ira & $\mathrm{N}$ & 1,10 & 1,80 & 20 & $-2,444$ & 0,019 & $0,1 /$ \\
\hline Pres mod & EQ & 6,95 & 2,01 & 20 & 1104 & 0276 & -035 \\
\hline Pres_mod & $\mathrm{N}$ & 6,25 & 2,00 & 20 & 1,104 & 0,210 & $-0,35$ \\
\hline Pres for & EQ & 0,55 & 1,67 & 20 & -0221 & 0834 & 0.07 \\
\hline & $\mathrm{N}$ & 0,65 & 1,31 & 20 & & & \\
\hline I in & EQ & 5,35 & 2,89 & 20 & -0483 & 0632 & 015 \\
\hline Lin_con & $\mathrm{N}$ & 5,80 & 3,00 & 20 & $-0,483$ & 0,032 & 0,15 \\
\hline Lin des & EQ & 0,60 & 1,23 & 20 & -0.115 & 0.909 & 0.04 \\
\hline LIn_ues & $\mathrm{N}$ & 0,65 & 1,50 & 20 & $-U, \perp \perp J$ & 0,909 & 0,04 \\
\hline Iin tre & EQ & 0,60 & 1,31 & 20 & 0171 & 0628 & 5 \\
\hline & $\mathrm{N}$ & 0,40 & 1,35 & 20 & $0,4 / 4$ & 0,638 & $-0,15$ \\
\hline Iin ref & EQ & 1,45 & 2,35 & 20 & 0 & 1000 & 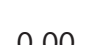 \\
\hline Lin_reI & $\mathrm{N}$ & 1,45 & 2,28 & 20 & 0 & 1,000 & 0,00 \\
\hline rep dup & EQ & 0,75 & 1,33 & 20 & 0396 & 0694 & -013 \\
\hline & $\mathrm{N}$ & 0,60 & 1,05 & 20 & & & \\
\hline ren fer & EQ & 0,45 & 0,83 & 20 & -0 82? & 0416 & 026 \\
\hline rep_iec & $\mathrm{N}$ & 0,70 & 1,08 & 20 & $-0,8<2$ & 0,416 & 0,26 \\
\hline Sea rig & EQ & 0,90 & 2,45 & 20 & 1547 & 130 & -049 \\
\hline seq_rig & $\mathrm{N}$ & 0,05 & 0,22 & 20 & 1,כ4/ & 0,130 & $-0,49$ \\
\hline Sea ord & EQ & 0,10 & 0,31 & 20 & -2536 & 0015 & 080 \\
\hline & $\mathrm{N}$ & 2,05 & 3,43 & 20 & $-2,536$ & 0,015 & 0,80 \\
\hline
\end{tabular}


Tabela 3 (continuação)

Estatísticas Descritivas, Teste t de Student e d de Cohen para os Grupo de Esquizofrênicos (EQ) e Normativo (N)

\begin{tabular}{|c|c|c|c|c|c|c|c|}
\hline Var. & Grupo & $M$ & $D P$ & $N$ & $t$ & $p$ & $d$ \\
\hline \multirow{2}{*}{ Seq_rel } & EQ & 3,10 & 3,71 & 20 & \multirow{2}{*}{0} & \multirow{2}{*}{1,000} & \multirow{2}{*}{0,00} \\
\hline & $\mathrm{N}$ & 3,10 & 3,71 & 20 & & & \\
\hline \multirow{2}{*}{ Tit_nom } & EQ & 6,30 & 2,34 & 20 & \multirow{2}{*}{0,186} & \multirow{2}{*}{0,853} & \multirow{2}{*}{$-0,06$} \\
\hline & $\mathrm{N}$ & 6,15 & 2,74 & 20 & & & \\
\hline \multirow{2}{*}{ Tit_exp } & EQ & 0,50 & 1,05 & 20 & \multirow{2}{*}{$-1,934$} & \multirow{2}{*}{0,061} & \multirow{2}{*}{0,61} \\
\hline & $\mathrm{N}$ & 1,75 & 2,69 & 20 & & & \\
\hline
\end{tabular}

Acerca dos códigos de qualidade formal, a diferença era esperada de acordo com a descrição clínica da esquizofrenia que, de forma geral, caracteriza-se pela alteração no contato com a realidade ligado aos aspectos perceptivos (American Psychological Association, 2002). É possível observar que para $\mathrm{FQu}$, o grupo normativo apresentou maior média e, consequentemente, para FQ-, o grupo critério ficou com média superior, indicando má adequação do desenho realizado em relação ao estímulo inicial. Pianowski e Villermor-Amaral (2010) e Villemor-Amaral et al. (2005) observaram resultados semelhantes em estudos, utilizando o Rorschach, ou seja, indicadores de testes baseados na percepção dos respondentes parecem mais sensíveis em patologias com princípio de distorção perceptiva.

O código de movimento humano indica forte conteúdo projetivo, visto que, como salientam Exner e Sendin (1999), esse é o único atributo que as manchas de tinta não possuem, senido assim, carregam grande carga projetiva do sujeito. Visto que o grupo normativo apresentou média superior, esse achado corrobora a intepretação proposta por Mihura, Meyer e Dumitrascu (2013), sinalizando que movimento humano indica planejamento, imaginação e empatia. Por fim, sobre a pressão fraca do lápis e a realização do desenho em sequência ordenada, podem estar relacionados ao processo motor preservado e à organização cognitiva, respectivamente.
Ainda na Tabela 3, é possível notar, para as variáveis com diferença significativas, os valores para o $d$ de Cohen, indicando diferenças expressivas, variando de 0,77 à 1,37. Por se tratar de uma técnica que tem por base avaliativa o processo perceptivo (Koffka, 1975; Kolck, 1974) esses resultados eram esperados visto que a esquizofrenia é caracterizada principalmente pela alteração no contato com a realidade, ou seja, relaciona-se ao continuum de apercepção (APA, 2002; Silva, 1989). Diferenças semelhantes a essa foram encontradas por diversos autores, indicando essa característica particular dos indivíduos com diagnóstico de esquizofrenia (Franco \& Villemor- Amaral, 2012; Hilsenroth, Fowler, \& Padawer, 1998; Meyer, 1993; Nascimento, 2006; Villemor Amaral et al., 2005).

Nesse sentido, estes resultados indicam que, por meio do sistema proposto por Pessotto (2015) para o Teste de Wartegg, foi possível diferenciar os grupos de acordo com o critério clínico utilizado, indicando a presença de evidência favorável. As evidências de validade devem ser verificadas de forma cumulativa e, de acordo com o grau em que aconteçam, possam sustentar as hipóteses propostas pelo instrumento no processo de avaliação psicológica (Urbina, 2007).

Com o objetivo de identificar a probabilidade de acertos no diagnóstico de esquizofrenia, empregou-se a regressão logística para as variáveis com diferença significativa. Os resultados podem ser visualizados na Tabela 4.

Tabela 4

Análise de Regressão Logística

\begin{tabular}{lccccccc}
\hline & $B$ & Erro Padrão & Wald & $d f$ & $p$ & Exp(B) & $R^{2}$ Cox \& Snell \\
\hline FQu & 0,476 & 0,183 & 6,796 & 1 & 0,009 & 1,61 & 0,274 \\
FQ- & 0,452 & 0,183 & 6,083 & 1 & 0,014 & 1,572 & 0,252 \\
CP_M & 2,234 & 0,859 & 6,757 & 1 & 0,009 & 9,339 & 0,233 \\
CP_FM & 21,426 & 20096,485 & 0 & 1 & 1,00 & 2019343554 & 0,139 \\
CP_p & 21,403 & 9934,417 & 0 & 1 & 1,00 & 1973852980 & 0,386 \\
Pres_fra & 1,267 & 0,664 & 3,64 & 1 & 0,056 & 3,549 & 0,185 \\
Seq_ord & 0,67 & 0,566 & 1,401 & 1 & 0,237 & 1,953 & 0,172 \\
\hline
\end{tabular}

De acordo com os resultados, é possível observar que as variáveis boa qualidade formal (FQu), má qualidade formal (FQ-) e movimento humano (M) tiveram valores significativos na predição do grupo. Dessas variáveis, destacam-se as ligadas à qualidade formal, retomando a hipótese sobre a percepção para o grupo de 
esquizofrênicos, bem como àquela relativa ao agrupamento, indicando um conjunto de variáveis útil na probabilidade de acerto do grupo. A partir desse resultado, buscou-se verificar a relação entre essas variáveis por meio da correlação de Pearson. Essa análise justifica-se a fim de observar se as variáveis estariam mensurando traços latentes semelhantes e, nesse caso, observar-se-ia, alta correlação. O resultado pode ser observado na Tabela 5.

Tabela 5

Correlação de Pearson entre Conjunto de Variáveis do Teste de Wartegg

\begin{tabular}{lllllll}
\hline & FQ- & FQu & M & FM & $p$ & Pres_fra \\
\hline FQu & $-0,98^{* *}$ & & & & & \\
M & $-0,30$ & $0,31^{*}$ & & & & \\
FM & $-0,22$ & 0,23 & $-0,43^{* *}$ & & & \\
p & $-0,27$ & 0,27 & $0,32^{*}$ & $-0,04$ & & \\
Pres_fra & $-0,26$ & 0,28 & 0,01 & 0,09 & 0,01 & \\
Seq_ord & $-0,08$ & 0,07 & 0,23 & $-0,1$ & 0,31 & $-0,13$ \\
\hline
\end{tabular}

Nota. ${ }^{* *}$ A correlação é significativa no nível 0,$01 ;{ }^{*}$ A correlação é significativa no nível 0,05

É possível notar quatro correlações significativas. Os valores encontrados entre boa qualidade formal $(\mathrm{FQu})$ e movimento humano (M) sendo 0,31, movimento humano (M) e movimento animal (FM) sendo $-0,43$ e movimento humano (M) e movimento passimo (p) sendo 0,32 , indicam correlações fracas, sinalizando que há covariância entre variáveis. Respectivamente essas correlações indicam que sujeitos que tendem a apresentar qualidade formal incomum, no sentido de pouco frequente, mas ainda ajustado à realidade, tendem a confeccionar figuras com movimento humano. Já pessoas que incluem em seus desenhos movimento humano, tentem a não inserir movimento animal. E, por fim, àqueles que realizam desenho com movimento humano, tendem a fazer, também, movimentos passivos.
Entre os códigos de qualidade formal, FQ- e FQu $(-0,98)$ a magnitude indica alta correlação negativa, sinalizando que as duas variáveis avaliam praticamente o mesmo traço latente, sendo este a adequação ou não à realidade acessada via percepção, isso se dá, pois os critérios acabam sendo empregados para avaliar dois extremos do continnum da percepção (Silva, 1989). Esse resultado é mais um indício do bom ajustamento da variável, além da diferença entre grupos verificada no teste $t$ de Student.

Optou-se, então, por verificar o melhor modelo, constituído de conjunto de variáveis, para a predição da esquizofrenia. É possível observar na Tabela 6 que o melhor modelo observado foi o constituído por duas variáveis, a saber, FQ- e M.

Tabela 6

Regressão Logística

\begin{tabular}{lccccccc}
\hline & $B$ & Erro Padrão & Wald & $d f$ & $p$ & Exp(B) & $R^{2}$ Cox \& Snell \\
\hline FQ- & $-0,386$ & 0,181 & 4,55 & 1 & 0,033 & 0,680 & 0,358 \\
M & 1,925 & 0,940 & 4,193 & 1 & 0,041 & 6,852 & \multirow{2}{*}{. } \\
\hline
\end{tabular}

A análise apresentada na Tabela 6 indica que o uso conjunto das variáveis FQ- e M pode ser útil no emprego para o diagnóstico da esquizofrenia, auxiliando no diagnóstico de 35,8\% dos casos. Sobre a qualidade formal, vários autores (Cardoso, 2012; Marques, Chaves, \& Yazigi, 2012; Mihura et al., 2013; Pianowski \& Villemor-Amaral, 2010; Scortegagna \& Villemor-Amaral, 2009) verificaram sua importância no que se refere à identificação de modos de percepção na utilização do Rorschach, o que corrobora com o presente resultado.

Sobre o movimento humano, Mihura et al. (2013), explanando sobre os indicadores das variáveis do Rorschach (R-PAS), relata que esse código, associado a uma má qualidade formal (M-), indica percepção distorcida de terceiros, podendo incluir distúrbios psicóticos. Essa ponderação parece justificar o resultado encontrado, salientando o caráter unificado das variáveis no diagnóstico da patologia. Franco e Villemor-Amaral (2012), em um estudo sobre a constelação PTI, também sinalizam M- enquanto indicador de perda de contato com a realidade relacionado a processos ideacionais desajustados.

Resende e Argimon (2012), em estudo com o Rorschach, contando com 80 sujeitos com diagnóstico de esquizofrenia, também verificaram a presença de movimento humano com qualidade formal menos de forma acentuada nos sujeitos. As autoras relatam que essa 
variável está associada a pensamentos distorcidos e fuga da realidade, podendo estar relacionado ainda à baixa habilidade social. Também Exner e Sendin (1999) salientam que a presença dessa variável nos protocolos, indicam pensamentos delirantes, mesmo que em pequena quantidade.

Frente a esse resultado, empregou-se, por fim, o procedimento curva ROC para verificar a capacidade discriminativa, bem como a sensibilidade e especificidade com o objetivo de verificar a acurácia diagnóstica desse agrupamento, a saber, Movimento Humano com Má Qualidade Formal (M-). O valor predito do agrupamento realizado a partir das duas variáveis foi verificado por meio de regressão logística. O resultado é apresentado na Figura 1.

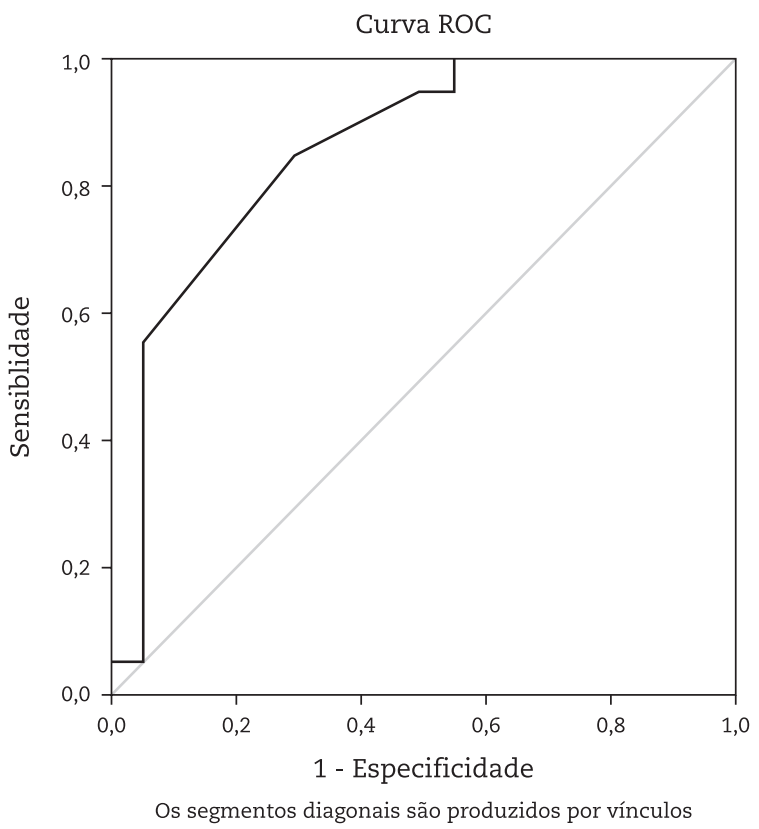

Figura 1. Curva ROC para W_FQ-_M

É possível observar, na Figura 1, bom ajustamento das variáveis, sendo que má qualidade formal (FQ-) apresentou um valor de área sob a curva de 0,788 , enquanto movimento humano (M), o valor de 0,855 . Além disso, as duas variáveis indicaram sensibilidade de $95 \%$ e especificidade de 55\%. A sensibilidade apresenta uma estimativa de verdadeiros positivos, enquanto a especificidade, de verdadeiros negativos (Martinez, LouzadaNeto, \& Pereira, 2003).

\section{Considerações Finais}

O presente estudo teve como objetivo verificar evidências de validade de critério para o conjunto de variáveis proposto por Pessotto (2015) para o Teste de Wartegg. Pode-se verificar que, do conjunto de variáveis propostas, algumas tiveram médias significativamente diferentes para os dois grupos estudados. Como é possível notar, para algumas variáveis, não observou-se diferença significativas entre os grupos. Embora o objetivo desse estudo não englobe todas as variáveis, ressalta-se duas questões ligadas a esses resultados. Primeiro, trata-se de um estudo preliminar, da criação de um novo sistema, portanto, muitas variáveis precisarão ser mais estudas ou, ainda, podem ter diferenças entre outros grupos, e não apenas esquizofrênicos. Segundo o foco foi utilizar as análises das variáveis que tradicionalmente são indicadas como bons indicadores para quadros de esquizofrenia na técnica de Rorschach. Contudo, optou-se por apresentar todas as variáveis, uma vez que poderia auxiliar na compreensão do sistema proposto. Sobre a probabilidade preditiva dessas variáveis, algumas demonstraram-se indicadas para tal objetivo, com destaque à qualidade formal menos (FQ-) e movimento humano (M).

Ressalta-se que este é um estudo inicial com o sistema, com isso, embora alguns índices tenham se demonstrado positivos, outros estudos são necessários para o desenvolvimento e consequente utilização do teste. Nesse sentido, utilizou-se o Rorschach como parâmetro de correlação e indicativos de variáveis, sabendo-se, contudo, que a natureza das tarefas são diferentes. A opção pelo Rorschach se deu pela sua grande amplitude de utilização e estudos, ressaltando-se novamente que se trata de um estudo inicial de um novo sistema de codificação e interpretação para Teste de Wartegg e novos estudos deverão 
ainda ser realizados. Estudos com outras fontes de informação, como evidências de validade baseadas na relação com outras variáveis, utilizando-se de instrumentos com maior tradição ou ainda com um número mais expressivo de participantes podem auxiliar no desenvolvimento do sistema.

\section{Referências}

Alves, I. C. B. (2008). Pesquisas com o teste de Wartegg no Brasil. Em: A. E. Villemor-Amaral \& B. S. G. Werlang (Orgs.), Atualizações em métodos projetivos para avaliação psicológica (pp. 345-363). São Paulo: Casa do Psicólogo.

American Psychological Association. (2002). Diagnostic and statistical manual of mental disorders, fourth edition: DSM-IV-TR ${ }^{\circledR}$. American Psychiatric Association.

Baumeister, R., \& Tice, D. M. (1988). On the stability of variability: Retest reliability of metratraits. Personality and Social Psyvhoogy Bulletin, 17(6), 633-639. doi: /10.1177/0146167291176005

Bornstein, R. F. (2001). Clinical utility of the rorschach inkblot method: Reframing the debate. Journal of Personality Assessment, 77 (1), $39-47$. doi: 10.1207/S15327752JPA7701_03

Bornstein, R. F. (2002). A process dissociation approach to objective-projective test score Interrelationships. Journal of Personality Assessment, 78(1), 47-68. doi: 10.1207/S15327752JPA7801_04

Campos, R. C. (2013). Além dos números há uma pessoa: Sobre a utilização clínica de testes. Avaliação Psicológica, 12(3), 291-298. Recuperado de http://pepsic.bvsalud.org/scielo.php?script=sci_arttext\&pid=S1677-04712013000300003

Cardoso, L. M. (2012). Comparação da sensibilidade de três Tistas de qualidade formal para avaliação de psicopatologia no Rorschach (Tese de Doutorado não publicada). Universidade São Francisco, Itatiba.

Chaplin, W. F. (1991). The next generation of moderator research in personality psychology. Journal of Personality, 59(2), 143-178. Recuperado de https:/www.ncbi.nlm.nih.gov/pubmed/1880698

Conselho Federal de Psicologia - CFP. Resolução no 002/2003 de 24 de março (2003).

Cunha, J. A. \& Nunes, M. L. T. (1993). Teste das Fábulas: forma verbal e pictórica. São Paulo: Centro Editor de Testes e Pesquisas em Psicologia.

Exner, J. E., \& Sendin, C. (1999). Manual de interpretação do Rorschach - Para o sistema compreensivo. São Paulo: Casa do Psicólogo.

Fensterseifer, L., \& Werlang, B. S. G. (2008). Apontamentos sobre o status científico das técnicas projetivas. Em A. E. Villemor-Amaral \& B. S. G. Werlang (Eds.), Atualizações em Métodos Projetivos para Avaliação Psicológica (pp. 15-33). São Paulo: Casa do Psicólogo.

Franco, R. R. C., \& Villemor-Amaral, A. E. de. (2012). O Zulliger e as constelações do Rorschach no sistema compreensivo. Avaliação Psicológica, 11(1), 141-152. Recuperado de http://pepsic.bvsalud.org/scielo.php?script=sci_arttext\&pid=S1677-04712012000100013

Freitas, A. M. L. (1993). Guia de Aplicação e Avaliação do Teste Wartegg. São Paulo: Casa do Psicólogo.

Garb, H. N., Wood, J. M., Lilienfeld, S. O., \& Nezworski, M. T. (2002). Effective use of projective techniques in clinical practice: Let the data help with selection and interpretation. Professional Psychology: Research and Practice, 33(5), 454-463. doi: 10.1037/0735-7028.33.5.454

Gronnerod, J. S., \& Gronnerod, C. (2012). The Wartegg Zeichen Test: A literature overview and a meta-analysis of reliability and validity. Psychological Assessment, 24(2), 476-489. doi: 10.1037/a0026100

Hilsenroth, M. J., Fowler, J. C., \& Padawer, J. R. (1998). The Rorschach schizophrenia index (SCZI): An examination of reliability, validity, and diagnostic efficiency. Journal of Personality Assessment, 70(3), 514-534. doi: 10.1207/s15327752jpa7003 9

Koffka, K. (1975). Princípios de Psicologia da Gestalt. São Paulo: Cultrix.

Kolck, O. L. V. (1974). Técnicas de exame psicológico e suas aplicações no Brasil. Vozes.

Lilienfeld, S. O., Wood, J. M., \& Garb, H. N. (2000). The scientific status of projective techniques. Psychological Science in the Public Interest, 1(2), 27-66. doi: 10.1111/1529-1006.002

Marques, T. C., Chaves, A. C., \& Yazigi, L. (2012). Estudo parcial da validação do atlas do Rorschach sistema compreensivo em amostra de pacientes psiquiátricos de São Paulo. Psico-USF, 17(3), 417-416. doi: 10.1590/S1413-82712012000300008.

Martinez, E. Z., Louzada-Neto, F., \& Pereira, B. de B. (2003). A Curva ROC para testes diagnósticos. Cadernos Saúde Coletiva, Rio de Janeiro, 11(1), 7-31. doi: 10.1590/S1413-82712012000300008

Meyer, G. J. (1993). The impact of response frequency on the Rorschach constellation indices and on their validity with diagnostic and MMPI-2 Criteria. Journal of Personality Assessment, 60(1), 153-180. doi: 10.1207/s15327752jpa6001_13

Meyer, G. J., \& Handler, L. (1997). The ability of the Rorschach to predict subsequent outcome: A meta-analysis of the Rorschach prognostic rating scale. Journal of Personality Assessment, 69(1), 1-38. doi: 10.1207/s15327752jpa6901_1

Meyer, G. J., \& Kurtz, J. E. (2006). Advancing personality assessment terminology: Time to retire "objective" and "projective" as personality test descriptors. Journal of Personality Assessment, 87(3), 223-225. doi: 10.1207/s15327752jpa8703_01

Mihura, J. L., Meyer, G. J., \& Dumitrascu, N. (2013). The Validity of Individual Rorschach Variables: Systematic Reviews and MetaAnalyses of the Comprehensive System. Psychological Bulletin, 139(3), 548-605. doi: 0.1037/a0029406

Montagna, M. E. (1989). Análise e interpretação do CAT: Teste de apercepção temática infantil. EPU.

Nascimento, R. S. G. F. do. (2006). Estudo normativo do sistema compreensivo do Rorschach para São Paulo: Resultados dos índices PTI, SCZI, DEPI, CDI, HVI, OBS e S-CON. Avaliação Psicológica, 5(1), 87-97. Recuperado de http://pepsic.bvsalud.org/scielo. php?script $=$ sci arttext\&pid $=$ S1677-04712006000100010

Pessotto, F. (2015). Teste de Wartegg (Sistema em Desenvolvimento). Itatiba: Laboratório de Avaliação Psicológica e Educacional (LabAPE) Universidade São Francisco (USF).

Pianowski, G., \& Villemor-Amaral, A. E. de. (2010). Location and formal quality of the Rorschach-SC in Brazil: Validity with non-patient sample. Psico-USF, 15(3), 333-343. doi: 10.1590/S1413-82712010000300007

Ramon, R. R. (2006). Wartegg: Precisão entre avaliadores e evidência de validade com o método de Rorschach (Dissertação de Mestrado). Universidade São Francisco, Itatiba. 
Resende, A. C., \& Argimon, I. I. L. (2012). A técnica de Rorschach e os critérios da CID-10 para o diagnóstico da esquizofrenia. Psicologia: Reflexão E Crítica, 25(3), 422-434. doi: 10.1590/S0102-79722012000300002

Roivainen, E. (2009). A Brief History of the Wartegg Drawing Test. Gestalt Theory, 31(1), 55-71. Recuperado de https://pdfs.semanticscholar. org/6a82/4783de7fc454d305e07fab1b9d9c957a8cfc.pdf

Scortegagna, S. A., \& Villemor-Amaral, A. E. (2009). Autopercepção no Rorschach de vítimas de abuso sexual infantil. Psico, 40(3), $328-336$. Recuperado de http://revistaseletronicas.pucrs.br/ojs/index.php/revistapsico/article/view/5736

Silva, M. C. V. (1989). TAT: Aplicação e interpretação do Teste de Apercepção Temática. São Paulo: EPU.

Silva, M. C. V. (2008). O teste de completamento de desenhos de Wartegg (WZT). Em A. E. Villemor-Amaral \& B. S. G. Werlang (Eds.), Atualizações em métodos projetivos para avaliação psicológica (pp. 339-344). São Paulo: Casa do Psicólogo.

Souza, C. V. R., Primi, R., \& Miguel, F. K. (2007). Validade do Teste Wartegg: Correlação com 16PF, BPR-5 e desempenho profissional.Avaliação Psicológica, 6(1), 39-49. Recuperado de http://pepsic.bvsalud.org/scielo.php?script=sci_arttext\&pid=S1677-04712007000100006

Swann, W. B., \& Seyle, C. (2005). Personality psychology's comeback and its emerging symbiosis with social psychology. Personality and Social Psychology Bulletin, 31 (2), 155-165. doi: 10.1177/0146167204271591

Urbina, S. (2007). Fundamentos da Testagem Psicológica. Porto Alegre: Artmed.

Villemor-Amaral, A. E. de. (2008). A validade teórica em avaliação psicológica. Psicologia: Ciência E Profissão, 28(1), 98-109. doi: 10.1590/ S1414-98932008000100008

Villemor-Amaral, A. E., \& Pasqualini-Casado, L. (2006). A cientificidade das técnicas projetivas em debate. Psico-USF, 11(2), 185-193. doi: 10.1590/S1413-82712006000200007

Villemor Amaral, A. E., Primi, R., Franco, R. R. C., Farah, F. H. Z., Cardoso, L. M., \& Silva, T. C. (2005). O teste de Pfister e sua contribuição para diagnóstico da esquizofrenia. Revista Do Departamento de Psicologia - UFF, 17(2), 89-98. doi: 10.1590/S0104-80232005000200008

Villemor-Amaral, A. E., \& Werlang, B. S. G. (2008). Atualizações em métodos projetivos para avaliação psicológica. Casa do Psicólogo.

Werlang, B. S. G. (2002). TAT, conforme o modelo de Bellak. Em J. A. Cunha (Ed.), Psicodiagnóstico-V (pp. 409-415). Porto Alegre: Artmed.

Werlang, B. S. G., \& Cunha, J. A. (1993). Avaliação da personalidade sob enfoque projetivo. In Psicodiagnóstico-R (pp. 123-129). Porto Alegre: Artes Médicas.

Wilson, T. D., Lindsey, S., \& Schooler, T. Y. (2000). A model of dual attitudes. Psychological Review, 107(1), 101-126. doi: 10.1037/0033295X.107.1.101

\section{Sobre os autores}

Fernando Pessotto é psicólogo, mestre e doutor em Psicologia pelo Programa de Pós-Graduação Stricto Sensu em Psicologia na Universidade São Francisco - área de concentração em Avaliação Psicológica, coordenador do Laboratório de Psicodiagnóstico e Neurociências Cognitivas (LaPeNC) e editor associado da Revista Sul Americana de Psicologia.

Ricardo Primi é psicólogo, doutor em Psicologia Escolar e do Desenvolvimento Humano pela Universidade de São Paulo com parte desenvolvida na Yale University (EUA), coordenador do Laboratório de Avaliação Psicológica e Educacional (LabAPE) e professor associado do Programa de Pós-Graduação em Psicologia da Universidade São Francisco (mestrado e doutorado em Avaliação Psicológica). 